\title{
Elusive Statistical Property of Arrival Rate and Holding Time used in Mobile Communication Networks
}

\author{
Osahenvemwen O.A., \\ Department of Electrical and \\ Electronics \\ Faculty of Engineering and \\ Technology. \\ Ambrose Alli, University. \\ Ekpoma, Edo State, Nigeria.
}

\author{
Edeko F.O. \\ Department of Electrical and \\ Electronics \\ Faculty of Engineering and \\ Technology. \\ University of Benin. \\ Benin, Edo State, Nigeria
}

\author{
Emagbetere J. \\ Department of Elect/Elect \\ Engineering \\ Faculty of Engineering and \\ Technology. \\ University of Benin \\ Benin City, Edo State.
}

\begin{abstract}
This research work is aimed at the study of arrival rate and holding time used in mobile communication networks, also to determine the best suitable statistical probability distribution of both arrival rate and holding time or service time in mobile communication network. The most general acceptable assumption about arrival rate is Poisson distribution and the holding time is exponential distribution in traffic modeling of mobile communication networks. Exhaustive literature review is deployed for details explanation on discrete random variables of arrival rate and continuous holding time use in traffic modeling of mobile communication networks. From the research work, the arrival rate is explained using point process or counting process, which leads to two unique properties, they are orderly and memorylessness. These unique properties are possessed by Bernoulli process with is discrete time, having Geometric distribution function, also with Poisson process, which is continuous time and discrete space, having Exponential distribution function which is used to characterize arrival rate based on interarrival rate process. Therefore, from the research work, it is assumed that arrivals rate is Poisson distribution and service time or holding time is exponentially distributed in traffic situation in mobile communication networks. These statistical properties since to the best suitable in mobile communication networks because of their unique parameters and are simple to analyses.
\end{abstract}

KEYWORDS: Random process, exponentially distribution, interarrival time, orderly and memoryless.

\section{INTRODUCTION}

The usages of basic infrastructures have created comfort for human existence on earth. These basic infrastructures have melted human aimed and aspiration by creating comfort on earth. Such basic infrastructures are Roads, Airports, Seaports, Banking system, Telecommunication system etc. The issue of usage and crowed control on these basic infrastructures are very important. The major problem arising from all these modern infrastructures is the issue of management, base on high population trying to gain access or want to use the infrastructures at a particular point in time. Sometime these modern infrastructures are over used due to limited capacity (channels). However, two possible effects occur, either there is a totals collapse of the infrastructure system or some customers will be frustrated, due to access denied. Therefore, the management and usage of modern infrastructures are very important, especially in view to obtain good Quality of Service (QOS), which is a major desire of all customers. The traffic usage of telecommunication infrastructures are carefully study in this research work. Subscribers wish to receive maximum quality of service from telecommunication operators, at any time he or she initiate calls and received calls. The telecommunication system consists of limited transmission channels or capacity, use to serve some volume of subscribers at a particular point in time. The manner at which subscribers uses these limited channels are random in nature, therefore these transmission channels are used or served in random process (Chi Wa et al., 2004; Hong et al.,1986 and Khan et al.,1997). Random process is one in which one or more quantities vary with time in such a way that the instantaneous values of quantities are not determined precisely but are predictable with certain probability, these quantities are known as random variables (Sanjay, 2010).

The subscriber's random usage of telecommunication infrastructure is divided into two segments such as arrival rate and holding time. The arrival process is the rate at which subscribers arrives at the service facility during a specified period of time. However, the arrival rate can be determined, by counting the number of subscribers during a specific period of time. Arrivals rate are assumed to be independent of each other and random over time. In mobile communication network, the arrival rate is referred to as the number of calls that will arrive at a switch (channel) during a finite period of time. The Greek letter lambda $(\lambda)$ is generally used to represent arrival rate (Georgeta et al.,2009;Zukerman,2010;Sanjay, 2010 and Kuumola et al.,2002). The holding time is referred to as the total time spent by the subscriber, using the channel or server (mobile equipment), from the commencement of service to the completion of the service. It also considered as the talk time or call length, call overhead time, plus queuing time if any. (Call duration and waiting time) the total time needed to serve a customer that required service in system. The holding time or service time is different from channel holding time, but both have the same statistical property. Channel holding time, this is the time a Mobile Station (MS) spend in a cell during a call (Georgeta et al.,2009;Alberto et al.,2008; http://www Cellular traffic-Wikipedia and Stefano et a.,12008).

The arrival rate and holding time are random variable which is trigger by the subscribers. This implied that subscribers use the expensive and limited channels or equipment in a random manner. A random variable is a real valued function defined on the sample space (Zukerman,2010; Balint et al.,2009).

There are basically two type of random variable; these are discrete random variable and continuous random variable. A random variable is called discrete if it takes at most a countable number of possible values or discrete probability $\mathrm{X}$ has only finite or countable infinity numbers of values. While 
a continuous random variable takes an uncountable number of possible values (Zukerman, 2010; Sanjay, 2010; Hong et al., 1986).

The well knows discrete probability distribution, that are used for statistical modeling includes; Poisson distribution, Bernoulli distribution, Binomial distribution, Geometric distribution and Negative binomial distribution or Pascal distribution( Zukerman 2010; Sanjay, S. 2010).

The continuous random variables are Uniform, exponentially, hyper-exponentially, Erlang, hypo-exponential Gaussian, multivariate Gaussian and Pareto (http://www.itu.int).

These are selected because of their applicability in teletraffic and related queuing models. Based on various research work, it shown that the exist queue models does take different format such as M/M/1, M/G/1, G/M/1 and G/G/1.In all these traffic models, M/M/1 traffic model is the most disciplined and simple means of evaluating the network performance in estimation of the queuing parameters, i.e. "Queue length" and "waiting time". In Erlang traffic model, it assumed Poisson distribution arrivals rate and exponentially distribution service time in traffic situation in mobile communication networks (Singh et al.,2007;Yuguang etal.,2005;Sanjay,2010 and Stefano et al.,2008)

\section{METHODOLOGY}

In this research work, it is focused on statistical probability distribution, which best represents arrival rate and holding time in mobile communication networks. The limitation of the study is on arrival rate and holding time or service time statistical properties used in mobile communication networks. Most often, queuing models are characterized by the Poisson arrival process, independent exponential service times and independence between the arrival process and the service times. They are denoted by $\mathrm{M}$ in the first two positions (i.e., $\mathrm{M} / \mathrm{M} /$. I.).Because of the memoryless property of Markovian chain, this is also amenable to analysis. Holistic approach is adopted to discover the relevant parameters involve, in the assumption of Poisson arrival rate and exponential service time in traffic modeling in mobile communication networks.

If the arrival rate is assumed to be Poisson distribution, therefore the Poisson random variable is discrete random variable, with a parameter $\lambda$ has the following probability function;

$p(x=i)=e^{-\lambda} \frac{\lambda^{i}}{i !} \quad \mathrm{i}=0,1,2,3 \ldots$

$\mathrm{P}=$ probability of subscriber,

A binomial random variable with parameters $n$ and $p$ approaches Poisson with parameter $\lambda=n p$.

A Poisson process is used to model random events as it emanating from a memoryless process. In Poisson process, the intervals between consecutive events are Independent and Identically Distributed (IID), which mean it is exponential distributed.

The exponential random variable has one parameter $\mu$ and its probability density function is given by.

$f(x)=\left\{\begin{array}{cc}\mu e^{-\mu x} & \text { if } x \geq 0 \\ 0 & \text { otherwise }\end{array}\right.$

(2)

Its distribution function is given by

$F(x)=\int_{0}^{x} \mu e^{-\mu s} d s=1-\mathrm{e}^{-\mu \mathrm{x}} \quad \mathrm{x} \geq 0$.

A convenient and useful way to describe the exponential random variable is by its complementary distribution function. It is given by,
$\bar{F}(X)=e^{-\mu X} \quad X \geq 0$

(4)

An important application of the exponential random variable is the time until the next call arrives at a switch or channel. This property is called the memoryless property of a random variable. A random variable is called memoryless if $p(x>s+/ x>t=p(x>s)$

(5)

If human lifetime were memoryless, then the probability that human being we survive at least 80 years given that we have survived 70 years is equal to the probability that a newborn baby lives to be 10 years. Human lifetime is not memoryless, but inter-arrival phone calls at the mobile communication switch or channel. To proof that exponential random variable is memoryless, using Equation 5 conditional probability definition together with the complementary distribution function of an exponential random variable as follows.

$$
\begin{aligned}
p\left(X>s+\frac{t}{X}>t\right) & =\frac{p(X>s+t \cap X>t)}{p(X>t)} \\
& =\frac{p(X>s+t)}{p(X>t)} \\
& =\frac{e^{-\mu(s+t)}}{e^{-\mu t}} \\
\text { (7) } & =e^{-\mu s}=p(x>s)
\end{aligned}
$$

\subsection{Statistical Property}

The arrival process, at the telecommunication exchange (switches) can be described using mathematical random point process or counting process. The description of point processes is as follows.

\subsubsection{Property of Point Process}

Point process has two basic properties, which includes;

- Orderliness

- Memorylessness

Orderliness is the probability that two or more arrivals happen at once are negligible. Mathematically, for a continuous -time counting process to be orderly, it should satisfy Equation 10 given as;

$\lim _{\Delta t \rightarrow o} P(X(t+\Delta t)-X(t)>1 / X(t+\Delta t)-X(t) \geq 1=$ $0(10)$

While memoryless is defined as, any point in time, the future evolution of the process is statistically independent of its past. These two unique properties are possessed by

$\checkmark$ Bernoulli process with discrete time, having Geometric distribution function.

$\checkmark$ Poisson process, with continuous time having Exponential distribution function.

These probability distribution properties can be explained using point process. Point process is a sequence of events or arrival calls which occurred at random point of time $t_{i}, i=1$, $2,3, \ldots \mathrm{t}_{\mathrm{i}+1}>\mathrm{t}_{\mathrm{i}}$

A point process can be defined by counting process $\{N(t), t \geq$ $0\}$, where $\mathrm{N}(\mathrm{t})$ is the number of arrivals occurred within $[0, \mathrm{t}]$. A counting process $\{N(t)\}$ has the following properties:

- $\quad \mathrm{N}(\mathrm{t}) \geq 0$,

- $\quad N(t)$ is integer,

- If $s>t$, then $N(s) \geq N(t)$ and $N(s)-N(t)$ is the number of occurrences within $[\mathrm{t}, \mathrm{s}]$.

Note that $\mathrm{N}(\mathrm{t})$ is not an independent process because for Example, if $t_{2}>t_{1}$ then $N\left(t_{2}\right)$ is dependent on the number of arrivals in $\left[0, t_{1}\right]$.

Another way to define a point process is by the stochastic process of the interarrival times 
$\Delta_{\mathrm{i}}=\mathrm{t}_{\mathrm{i}+1}-\mathrm{t}_{\mathrm{i}}$,

These values can be continuous or discrete consider to be real values or non negative values.

\subsection{Bernoulli Process}

The Bernoulli process is a discrete- time stochastic process made up of a sequence of Independent and Identical Distribution (IID) Bernoulli distributed random variables

$\left\{X_{i}, i=0,1,2,3, \ldots\right\}$. Where for all $i, p\left(X_{i}=1\right)=p$ and $p\left(X_{i}=0\right)=1-p$.

The Bernoulli process is both orderly and memoryless. It is orderly because, by definition, no more than one arrival can occur at any time-slot as the Bernoulli random variable takes values of more than one with probability zero. It is memoryless because the Bernoulli trails are independent, so at any discrete point in time $\mathrm{n}$, the future evolution of the process is independent of its past.

The counting process for the Bernoulli process is another discrete-time stochastic process $\{N(n), n \geq 0\}$ where is a sequence of Binomial random variable $N(n)$ representing the total number of arrivals occurring within the first $\mathrm{n}$ time-slots. Therefore, since will start from timeslot $0, \mathrm{~N}(\mathrm{n})$ does not include slot $\mathrm{n}$ in the counting. That is, given as

$$
p[N(n)=i]=\left(\begin{array}{c}
n \\
i
\end{array}\right) p^{i}(1-p)^{n-i} \quad i=1,2, \ldots, n .
$$

The concept of an interarrival time for the Bernoulli process can be explained as follows. Let assume without loss of generality that there was an arrival at time-slot $\mathrm{k}$, the interarrival time will be the number of slot between $\mathrm{k}$ and the first time-slot to have an arrival following $\mathrm{k}$. Time-slot $\mathrm{K}$ is not counted, while it will count the time-slot of the next arrival. Because the Bernoulli process is memoryless, the interarrival times are IID, so will can drop the index $\mathrm{i}$ of $\Delta_{\mathrm{i}}$, designating the interarrival time, and consider the probability function of the random variable $\Delta$ representing any interarrival time. Because $\Delta$ represents a number of Bernoulli trials until a success occurs, it is geometrically distributed, and its probability function is given by

$P(\Delta=i)=p(-p)^{i-1} \quad \mathrm{i}=1,2, \ldots$

$$
\text { (12) }
$$

\subsection{Poisson Process}

The Poisson process is a continuous - time point process which is also memoryless and orderly. A point process or counting process $\{\mathrm{N}(\mathrm{t})\}$ is used to explain Poisson process with rate $\lambda>0$ if it satisfies the following three condition.

1. $\mathrm{N}(0)=0$.

2. The numbers of occurrences in two non-overlapping intervals are independent. That is, for any $s>t>u>$ $\mathrm{v}>0$, the random variable $\mathrm{N}(\mathrm{S})-\mathrm{N}(\mathrm{t})$ and the random variable $\mathrm{N}(\mathrm{u})-\mathrm{N}(\mathrm{v})$ are independent. This means that the Poisson process has what is called independent increments.

3. The number of occurrences in an interval of length $t$ has a Poisson distribution with mean $\lambda$ t.

These three conditions are referred to as three Poisson conditions.

Poisson process has what is called stationary increment, that is, for any $t_{2}>t_{1}$, the random variable $\mathrm{X}\left(\mathrm{t}_{2}\right)-\mathrm{X}\left(\mathrm{t}_{1}\right)$, and random variable $X\left(t_{2}+u\right)-X\left(t_{1}+u\right)$ have the same distribution for any $\mathrm{u}>0$, also the distribution is Poisson with parameter $\lambda\left(\mathrm{t}_{1}-\mathrm{t}_{2}\right)$ with the time interval as $\Delta=\left(t_{2}-t_{1}\right)$. The chance of having occurrence is the same and occurrences are equally likely to happen at all times. This property is also called timehomogeneity.
Another important property of the Poisson process is that the inter-arrival times of occurrence is exponentially distributed with parameter $\lambda$. This is shown by considering $s$ to be an occurrence and $\mathrm{T}$ the time until the next occurrence, recall that $P(T>t)=P\left(N(t=0)=e^{-\lambda t} \quad, \quad\right.$ and recalling the properties of independent and stationary increments. As a result, the mean interarrival time is given by

$$
E[T]=\frac{1}{\lambda}
$$

By the memoryless property of the exponential distribution, the time until the next occurrence is always exponentially distributed and therefore, at any point in time, not necessary at points of occurrences, the future evolution of the Poisson process is independent of the past, and always probabilistically the same. The Poisson process is therefore memoryless. Actually, the independence of the past can be explained also by the Poisson process property of independent increments, which can also be explained by stationary increment property.

The random point in time, independent of a given Poisson process, the time until the next occurrence event has exponential distribution with parameter $\lambda$. Because process in reverse is also a Poisson process, then at any point in time, the time passed from the last Poisson occurrence event also has exponential distribution with parameter $\lambda$. therefore, if a random point is consider in teams of the mean length time interval between two consecutive Poisson occurrence must be $1 / \lambda+1 / \lambda=2 / \lambda$. The time between consecutive Poisson occurrences are exponentially distributed with mean $1 / \lambda$, which can be explained as a point of time at random is likely to pick an interval that is longer than the average.

In Table 1 provide the mean and the variance of some random variable

\begin{tabular}{|l|c|c|c|}
\hline $\begin{array}{l}\text { Random } \\
\text { variable }\end{array}$ & parameters & mean & variance \\
\hline Bernoulli & $0 \leq p \leq 1$ & $p$ & $p(1-p)$ \\
\hline Binomial & $n$ and $0 \leq p \leq 1$ & $n p$ & $n p(1-p)$ \\
\hline Poisson & $\lambda>0$ & $\lambda$ & $\lambda$ \\
\hline Uniform & $a$ and $b$ & $(a+b) / 2$ & $\left.(\sigma-1)^{2}\right) / 12$ \\
\hline Exponential & $\mu>0$ & $1 / \mu$ & $1 / \mu^{2}$ \\
\hline Gaussian & $m$ and $\sigma$ & $m$ & $\sigma^{2}$ \\
\hline pareto & $\begin{array}{l}\delta>0 \text { and } 1<\gamma \\
\leq 2\end{array}$ & $\frac{\delta \gamma}{\gamma-1}$ & $\infty$ \\
\hline
\end{tabular}

The Poisson process is characterized by one parameter $\lambda$ ant $\lambda t$ is the mean as well as the variance shown in Table 1,the number of occurrences during any time interval of length $t$, it is defined as a continuous-time process, possessed the memoryless property.

Services times are assumed mutually independent and are independent of the arrival process, and also independent of the interarrival times. The Services times or channel holding time is determined by the cell residence time (cell dwell time).The cell residence is affected by the mobile moving speed, geographical location of the Mobile Station (MS) (or other factors such as fading) etc. Channel holding time, it is the time a MS remains in the same cell during a call, while call holding time is the total call duration time, which involves the movement of MS from a cell to another new cell, that process is called handover process http://www Cellular traffic-Wikipedia).

Channel holding time, call holding time and interarrival time of a cell are all assumed to be exponential 
distribution. From research work, the assumption of exponential distribution in traffic situation is base on simple means of evaluating the network performance (Yuguang;2005;Singh et al 2007 ;Sanjay,2010 and Stefano et al.,2008).

Markov-chains are certain discrete space stochastic processes which are amenable for analysis in the traffic characterization, modeling of queuing and telecommunication networks systems are classified into two groups; Discrete time Markov-chains and continues Markov-chains.

\subsection{Markovian Systems}

If the probability of moving from one state to another depends on the present state only, and not on any previous state, the process is called a Markov chain. The common characteristic of all markovian systems is that all interesting distributions, namely the distribution of the interarrival times and the distribution of the service times are exponential distribution and thus exhibit the Markov (memoryless) property. Based on property, there are two factors

- The state of the system can be summarized in a single variable, namely the number of customer in the system.(If the service distribution is not memoryless, this is not longer true, since not only the number of subscribers in the system is needed, but also the remaining service time of the customer in service).

- Markovian systems can be directly mapped to a Continuous Time Markov Chain (CTMC) which can be easily solved.

In general, the aim of statistical probability is used to characterize the random variable in traffic modeling which provide the network designer with relatively simple means to represent traffic load on a mobile communication networks.

\section{CONCLUSION}

This research is focused on the determination of the best assumption in statistical property of arrival rate and holding time used in mobile communication networks. Different statistical properties are used in traffic modeling of network system such as Roads, Airports, Seaports, Banking system, Students registration in campus, Barber saloon etc. The subscribers or customers uses these available network facilities and traffic is been generated due to usage of these facilities. The traffic is made up of two basic parameters, they includes the arrival rate and holding time. These parameters are represented in statistical probability because they are random variables, for proper accuracy in analytical traffic models. The basic problem arises from which statistical probabilities distribution best suit these saturations of arrival rate and holding time in a mobile communication networks. It was deduced from the research, that arrival rate can be explained using point process or counting process, leads to orderly and memoryless property. These two unique properties are possessed by Bernoulli process with discrete time having geometric distribution function and Poisson process, with continuous time having exponential distribution function. While Poisson process, with is continuous time and discrete space, having exponential distribution function is best used to characterize arrival rate based on interarrival rate. The assumption of exponential distribution in traffic situation is based on simple means of evaluating the network performance. . Therefore, from the research work, it is assumed that arrivals rate is Poisson distribution and service time or holding time is exponentially distributed in traffic situation in mobile communication networks. These statistical properties since to the best suitable in mobile communication networks because of their unique parameters and are simple to analyses.

\section{REFERENCES}

[1] Alberto E .Garcia and Elaus D. Hackbarth (2008) ,Approximation to a Behavioral Model for Estimating Traffic Aggregation scenarios,Journal of Universal Computer Science,Vol.14,No.5,pp 732-744

[2] Balint C.,Budura G., Budura A., and Marza E.,(2009),Dimensioning Rules Regarding Radio Resources In GSM /GPRS Network, WSEAS Transactions on Communications, Issue 8, Vol. 8,pp822832.

[3] Chi Wa Leong, Weihua Zhuang, yu Cheng, and Lei Wang (2004), Call Admission Control for Integrated ON/OFF Voice and Best Effect Data Services In Mobile Cellular Communications, IEEE Transactions On Communications, Vol.52, No.5,pp 778-781

[4] Georgeta, B., Cornel, B., Adrian, B. and Eugen, M. (2009). "Traffic models and Associated parameters in GSM/(E)GPRS Networks"WSEAS Transaction on Communications,ISSN:1109-2742,Issue

[5] Hong D. and Rappaport S.(1986) Traffic model and performance analysis for cellular mobile radio telephone systems with prioritized and nonprioritized handoff procedures, IEEE Trans.Veh. Technol. Vol. VT-35, pp72-928, Volume 8,pp 833-841.

[6] Khan F. and Zeghalache D.,(1997),Effect of cell residence time distribution on the performance of cellular mobile networks, in Proc. IEEE VTC 97 Phoenix Pp949-953.

[7] Kuumola E., Resing ,J. Virtamo J. (2002),Joint Distribution of instantaneous and Average Queue length in an M/M/1/K System. In proceeding of the $15^{\text {th }}$ Teletraffic congress specialist seminar "internet Traffic Engineering and Traffic management". Wurzburg, Germany Pp58-67.

[8] Sanjay, S. (2010). Computer networks, $1^{\text {st }}$ Edition, S.K. Kataria and Sons, page 621-629.

[9] Singh L.K and Srivastava R. (2007) "Memory estimation of internet server using queuing theory:comparative study between $\mathrm{M} / \mathrm{G} / 1, \mathrm{G} / \mathrm{M} / 1 \& \mathrm{G} / \mathrm{G} / 1$ queuing model" world academy of science, engineering and Technology 33 page $153-157$

[10] Stefano B.,Roberto C.,and Maurizio D. (2008)“An Empirical study on time -correlation of GSM telephone traffic"IEEE Transactions on wireless communications, Vol. 7,No.9,pp3428-3435.

[11] Yuguang Fang(2005)"Modeling and Performance Analysis for wireless mobile networks:A New Analytical Approach" IEEE/ACM Transactions on Networking, Vol. 13,no. 5,pp 989-1002.

[12] Zukerman Moshe (2010) "Introduction to queue theory and stochastic teletraffic model"pp 67,87,104-127.

[13] http://www Cellular traffic-Wikipedia; Channeling holding time, page

[14]http://www.itu.int. "Teletraffic Engineering” International Telecommunication union page 68-69, 119-186, ITU-D 2/4/2010. 